\title{
Efeito das Drogas Anti-Retrovirais sobre as Taxas de Fertilidade de Ratas Wistar
}

\author{
Effects of Antiretroviral Drugs on Fertility of Wistar Rats
}

\begin{abstract}
Ernesto Antonio Figueiró Filho, Geraldo Duarte, Alzira Amélia Martins Rosa e Silva, Benedito Antonio Lopes da Fonseca, Marisa Márcia Mussi-Pinhata, Silvana Maria Quintana, Patrícia El Beitune
\end{abstract}

\section{RESUMO}

Objetivo: avaliar os efeitos de drogas anti-retrovirais isoladas e em associação sobre as taxas de fertilidade em ratas prenhes expostas a estes fármacos, bem como o efeito perinatal nas crias.

Métodos: foram utilizadas ratas fêmeas prenhes adultas da raça Wistar, pesando inicialmente entre 200 e $230 \mathrm{~g}$. Foram testadas a azidotimidina (AZT), lamivudina (3TC) e nelfinavir (NFV), cujas dosagens foram padronizadas em 10 vezes a dose normalmente utilizada em gestantes, proporcionalmente ao peso dos animais. No total foram avaliados sete grupos, incluindo o controle. O inicio da experimentação foi o dia zero da prenhez e as cesarianas foram realizadas com 21 dias de prenhez. Para o cálculo das taxas de fertilidade, avaliaram-se o número de fetos vivos e mortos, sítios de implantação e número de corpos lúteos. Utilizaram-se o teste $\mathrm{t}$ de Student e Mann-Whitney para a análise estatística.

Resultados: não houve alterações significativas nas taxas de perdas pré-implantação e da eficiência de implantação de ratas tratadas com anti-retrovirais isolados e em associação. No entanto, houve aumento significativo nas taxas de perdas pós-implantação (grupo controle: 7,6\%; grupos medicamentos: de 20,2 a 26,7\%), redução significativa nas taxas de viabilidade fetal (grupo controle: 92,4\%; grupos medicamentos: de 73,3 a 79,8\%) e redução significativa no número de fetos por ninhada (grupo controle: 14,7, grupos medicamentos: de 11,1 a 12,7). Observou-se também que houve redução do peso materno e dos fetos nos grupos tratados com 3TC, AZT + 3TC e AZT + 3TC + NFV.

Conclusão: com a utilização de doses elevadas de medicamentos anti-retrovirais na presente casuistica, efeitos importantes sobre a fertilidade foram encontrados, o que sinaliza para a necessidade de se pesquisarem outros fármacos anti-retrovirais com menor potencial histotóxico e que possam, com segurança, ser utilizados por gestantes portadoras da infecção pelo virus da imunodeficiência humana.

PALAVRAS-CHAVE: Drogas anti-retrovirais, infecções e gravidez. Fertilidade. AIDS. Estudo experimental.

Departamento de Ginecologia e Obstetrícia da Faculdade de Medicina de Ribeirão Preto da Universidade de São Paulo Trabalho realizado com apoio FAPESP (Processo $01 /$ 00084-2)

Correspondência:

Geraldo Duarte

Hospital das Clínicas da Faculdade de Medicina de Ribeirão Preto da Universidade de São Paulo

Avenida Bandeirantes, 3900

14049-900 - Ribeirão Preto - SP

Fone: (16) 602-2588

e-mail: gduarte@fmrp.usp.br

\section{Introdução}

A transmissão materno-fetal é a principal forma pela qual crianças são infectadas pelo vírus da imunodeficiência humana tipo 1 (HIV-1). A incidência de transmissão vertical do HIV-1 nos Estados Unidos da América aumentou a partir de 1984, tendo seu pico em 1992. Observou-se redução de $67 \%$ nesta forma de transmissão no período compreendido entre 1992 e 1997. A redução da 
transmissão vertical decorreu do uso da azidotimidina (AZT) em mães infectadas pelo HIV1, cuja utilização aumentou de 7\% em 1993 para 91\% em $1997^{1-3}$.

O primeiro ensaio clínico no qual se administrou agente anti-retroviral a gestantes para avaliação de sua segurança, tolerância e eficácia na redução da transmissão vertical do HIV-1 foi publicado em 1994 pelo Pediatric AIDS Clinical Trials Groups 076 (PACTG 076). Este trabalho concluiu que a administração de AZT a gestantes durante o período gestacional, durante o trabalho de parto, acrescida do uso dessa medicação ao recém-nascido, foi responsável por reduzir a transmissão vertical do vírus em $67,5 \%{ }^{1}$.

Os avanços obtidos a partir do entendimento da patogênese da transmissão perinatal do HIV1 e sua redução com o uso do AZT estimularam o estudo de novas drogas com potente ação antiretroviral que pudessem ser utilizadas com segurança no período gestacional. No entanto, são escassas as informações sobre doses, farmacocinética e efeitos colaterais desses fármacos durante a gestação $0^{4,5}$.

Adicionalmente, já se conhecem alguns efeitos adversos maternos e fetais com uso de alguns esquemas durante a gestação. Quanto à mãe, foram observados: anemia, elevação de aminotransferases, náuseas e vômitos, intolerância à glicose, nefrolitíase, diarréia, hipertensão e diabete melito $^{6,7}$. Os efeitos sobre o feto incluem: prematuridade, anemia, anasarca, anoftalmia, angioma cutâneo, criptorquidismo, costela supranumerária e hepatite transitória 7 . Vários estudos analisaram os efeitos das drogas anti-retrovirais sobre o binômio mãe-feto, testando sua eficácia e toxicidade, no sentido de facilitar e orientar os experimentos clinicos. Por questões éticas, os estudos baseiam-se em modelos experimentais ${ }^{8-11}$.

A maioria dos trabalhos experimentais encontrados na literatura faz uso de modelos animais de viremia, com ratos transgênicos ou com inoculações de oncovírus ou de vírus que induzem leucemia nesses animais, cujo objetivo é de mimetizar a infecção pelo HIV-1 em seres humanos. Tais animais infectados ou mutados geneticamente servem de modelo para testes de drogas anti-retrovirais, com a finalidade de avaliar seus efeitos terapêuticos, eficácia e efeitos colaterais $^{8-11}$. No entanto, em ampla revisão da literatura, constatou-se a escassez de estudos sobre a ação das drogas anti-retrovirais em animais não infectados, utilizando modelos experimentais de gestação. Até o presente, poucas informações são fornecidas a respeito do efeito das drogas anti-retrovirais e suas combinações sobre as taxas de fertilidade em ratas prenhes ${ }^{12-16}$.
Os efeitos de drogas anti-retrovirais já foram estudados em símios, analisando a passagem placentária desses fármacos, a redução da carga viral e os efeitos perinatais em suas crias ${ }^{17-19}$. Por sua vez, os efeitos pós-natais em fetos de ratas expostas a medicamentos anti-retrovirais no período de prenhez já estão bem estabelecidos, com amplo material presente nas principais fontes de pesquisa $^{20-24}$.

Dentre os parâmetros associados a fertilidade estudados, destacam-se as taxas de perdas pré e pós-implantação, taxas de eficiência de implantação e as taxas de viabilidade fetal. Inúmeros estudos apresentam resultados com alterações diversas nesses índices, com os mais diversos tipos de drogas, dentre eles alguns antiretrovirais ${ }^{12-16,20-24}$.

Como visto, existem lacunas no conhecimento do efeito das drogas anti-retrovirais sobre as taxas de fertilidade em animais e, até o presente, modelos experimentais para avaliar o efeito desses fármacos sobre as taxas reprodutivas em ratos durante a prenhez ainda não foram amplamente testados. Com base nessas informações, objetivou-se com o presente estudo estudar a ação de drogas anti-retrovirais, isoladas e em associação, sobre as taxas de fertilidade de ratas prenhes expostas a estes fármacos, bem como o prognóstico neonatal das crias.

\section{Métodos}

Estudo experimetal realizado no Biotério do Departamento de Clínica Médica da Faculdade de Medicina de Ribeirão Preto da Universidade de São Paulo (FMRP-USP). Foram utilizadas 70 ratas fêmeas prenhes adultas da raça Wistar, com peso inicial entre 200 e 230 g. Optou-se por trabalhar com os medicamentos anti-retrovirais mais utilizados durante a gestação: azidotimidina (AZT), lamivudina (3TC) e nelfinavir (NFV). Tais drogas foram fornecidas pela Farmácia do Hospital das Clínicas da FMRP-USP e suas dosagens foram padronizadas em 10 vezes a dose utilizada em mulheres grávidas, proporcionalmente ao peso dos animais. Desse modo, as ratas prenhes receberam doses de $25 \mathrm{mg} /$ dia de AZT; $12,5 \mathrm{mg} /$ dia de 3TC; $97,5 \mathrm{mg} /$ dia de NFV.

A via de administração dos anti-retrovirais foi oro-gástrica, utilizando a técnica de gavagem, após diluição dos medicamentos em $5 \mathrm{~mL}$ de solução fisiológica a $0,9 \%$. O grupo controle recebeu solução fisiológica na mesma proporção. A administração foi sempre pela manhã, em dose única diária. 
As ratas prenhes adultas foram divididas em sete grupos com 10 animais em cada, sendo um controle e seis grupos que receberam medicamentos anti-retrovirais isolados e em associação. Uma vez que o período de prenhez de ratos tem duração de 21 dias, a análise dos efeitos das drogas sobre o organismo dos animais e de suas crias foi realizada no $21^{\circ}$ dia de prenhez. A experimentação teve início no "dia zero" da prenhez de todos os animais de cada grupo. A prenhez foi diagnosticada pela análise microscópica do esfregaço vaginal, sendo a observação da presença de espermatozóides no esfregaço como indicador do "dia zero" da prenhez. Os animais receberam água e alimentação ad libitum antes e durante o período de prenhez. O peso dos animais foi aferido diariamente após diagnosticada a prenhez.

No $21^{\circ}$ dia de prenhez os animais foram sacrificados por decapitação e imediatamente submetidos a cesariana, a qual foi sempre realizada em prazo inferior a 5 minutos pós-morte. Uma vez aberto o útero, o qual foi retirado em bloco do ventre do animal, foram contados os fetos, que foram retirados para anotação do sexo, pesagem dos mesmos e respectivas placentas. Em seguida, os cornos uterinos foram dissecados e, por observação direta, anotada a presença de reabsorções precoces e tardias (abortamentos). As reabsorções tardias foram reconhecidas pela possibilidade de distinção entre os tecidos embrionários e placentários, o que não acontece na reabsorção precoce. O número de sítios de implantação foi obtido pela soma de fetos vivos, fetos mortos e reabsorções. Em seguida, os ovários foram removidos para contagem dos corpos lúteos (indicativos do número de ovulações), reconhecidos pela cor rósea e pela forma arredondada medindo cerca de $2 \mathrm{~mm}$.

Com os valores obtidos, foram efetuados os seguintes cálculos para análise da fertilidade, segundo critérios divulgados por Almeida et al. ${ }^{25}$ taxa de perdas pré-implantação: total corpos lúteos total de sítios de implantação x 100/total de corpos lúteos; taxa de eficiência de implantação: total de sítios de implantação x 100/total de corpos lúteos; taxa de perdas pós-implantação: total sítios implantação - total fetos vivos x 100/total de sítios de implantação; taxa de viabilidade fetal: total de fetos vivos x 100/total de sítios de implantação.

Para análise estatística de variáveis paramétricas com distribuição normal e variâncias semelhantes, como peso dos animais prenhes, número de fetos e peso dos fetos e placentas, foi utilizado o teste $t$ de Student para comparação entre dois grupos.

Para análise de variáveis não paramétricas, como as taxas de fertilidade dos animais, foi utilizado o teste de Mann-Whitney para comparação de dois grupos. Foram consideradas diferenças estatisticamente significativas quando o valor de "p" bicaudal foi menor que 0,05 .

\section{Resultados}

Quanto à variável ganho de peso ao final da prenhez, apenas os grupos com a presença do 3TC tiveram ganho de peso inferior ao grupo controle (Tabela 1). Com referência às taxas de fertilidade, não houve diferenças nas taxas de perdas préimplantação (TPP) e nas taxas de eficiência de implantação (TEI) ao final de 21 dias de prenhez, quando se comparou o grupo controle com os demais grupos de animais que receberam medicamentos anti-retrovirais.

Tabela 1 - Médias do ganho de peso materno diário e médias do peso dos fetos após 21 dias de prenhez.

\begin{tabular}{|c|c|c|c|c|}
\hline Grupo & $\begin{array}{c}\text { Ganho de peso materno }(\mathrm{g}) \\
\text { média + } 1 \text { DP }\end{array}$ & $p^{*}$ & $\begin{array}{l}\text { Peso fetos }(\mathrm{g}) \\
\text { média + } 1 \mathrm{DP}\end{array}$ & $\mathbf{p}^{*}$ \\
\hline Controle & $8,5 \pm 1,4$ & - & $6,1 \pm 0,6$ & - \\
\hline AZT & $7,7 \pm 1,5$ & n.s & $5,6 \pm 0,7$ & n.s \\
\hline 3TC & $5,9 \pm 2,9$ & 0,01 & $4,9 \pm 1,1$ & 0,01 \\
\hline NFV & $8,1 \pm 1,1$ & n.s. & $5,7 \pm 0,8$ & n.s. \\
\hline $\mathrm{AZT}+\mathrm{NFV}$ & $8,0 \pm 0,7$ & n.s. & $5,9 \pm 0,2$ & n.s. \\
\hline$A Z T+3 T C+N F V$ & $6,9 \pm 1,0$ & 0,009 & $5,6 \pm 0,4$ & 0,009 \\
\hline
\end{tabular}

$p<0,05 t$ de Student/Comparações: grupo controle $\mathrm{x}$ grupo medicamentos. n.s.: não significante 
Em relação às taxas de perdas pós-implantação (TPPI), ao final de 21 dias de prenhez, todos os grupos apresentaram TPPI com valores significativamente maiores em relação ao controle (grupo controle: $7,6 \%$; grupos medicamentos: de 20,2 a 26,7\%), (Tabela 2). Observando as taxas de viabilidade fetal (TVF), pode-se notar que foram significativamente menores em todos os grupos, quando comparados ao controle (grupo controle: 92,4\%; grupos medicamentos: de 73,3 a $79,8 \%$ ), após 21 dias de prenhez (Tabela 2).

Tabela 2 - Taxas de perdas pós-implantação (TPPI) e taxas de viabilidade fetal (TVF) após 21 dias de prenhez.

\begin{tabular}{lcccc}
\hline Grupo & $\begin{array}{c}\text { \% TPPI } \\
\text { (M P25-P75) }\end{array}$ & $\mathbf{p}^{*}$ & $\begin{array}{c}\text { \% TVF } \\
\text { (M P25-P75) }\end{array}$ & $\mathbf{p}^{*}$ \\
\hline Controle & 7,6 & - & 92,4 & - \\
AZT & $(6,7-21,9)$ & & $(78,1-93,2)$ & \\
& 26,7 & 0,002 & 73,3 & 0,002 \\
3TC & $(16,6-34,0)$ & & $(66,0-83,3)$ & \\
& 26,3 & 0,002 & 73,7 & 0,002 \\
NFV & $(23,0-62,5)$ & & $(37,5-77,0)$ & \\
& 24,3 & 0,005 & 75,6 & 0,005 \\
AZT + 3TC & $(15,4-29,4)$ & & $(70,6-84,5)$ & \\
& 24,2 & 0,03 & 75,7 & 0,03 \\
AZT + NFV & $(10,5-29,4)$ & & $(70,6-89,5)$ & \\
& 20,2 & 0,03 & 79,8 & 0,03 \\
AZT + 3TC + NFV & $(9,8-26,3)$ & & $(73,6-90,1)$ & \\
& 23,5 & 0,03 & 76,5 & 0,03 \\
& $(9,6-28,6)$ & & $(71,4-90,4)$ & \\
\hline
\end{tabular}

" $p<0,05$ Mann-Whitney/Comparações: grupo controle $x$ grupo medicamentos

Tabela 3 - Médias do número de fetos/animal após 21 dias de prenhez.

\begin{tabular}{lcc}
\hline Grupo & $\begin{array}{c}\text { Número fetos/animal } \\
\text { média + 1 DP }\end{array}$ & $\mathbf{p}^{*}$ \\
\hline Controle & $14,7 \pm 1,4$ & - \\
AZT & $12,1 \pm 2,3$ & 0,007 \\
$3 T C$ & $11,1 \pm 4,3$ & 0,02 \\
NFV & $12,7 \pm 2,2$ & 0,02 \\
AZT + 3TC & $11,5 \pm 2,4$ & 0,002 \\
AZT + NFV & $11,5 \pm 2,4$ & 0,002 \\
AZT + 3TC + NFV & $11,4 \pm 3,8$ & 0,02 \\
\hline p $<0,05 t$ de Student/Comparações: grupo controle $x$ grupo medicamentos &
\end{tabular}

Comparando o número de fetos por animal prenhe, observou-se que aos 21 dias de prenhez, todos os grupos com uso de fármacos anti- retrovirais apresentaram redução significativa do número de fetos por ninhada (grupo controle: 14,7; grupos medicamentos: de 11,1 a 12,7) (Tabela 3).

Após 21 dias de prenhez, observou-se redução significativa dos pesos dos fetos nos grupos 3TC, AZT + 3TC e AZT + 3TC + NFV (Tabela 1), não ocorrendo, no entanto, alterações significativas com os pesos das placentas.

\section{Discussão}

Conceitualmente, fecundidade é a capacidade para a reprodução, ao passo que fertilidade refere-se à concretização da reprodução e pode ser medida pelo produto final, ou seja, o número de novos indivíduos ${ }^{25}$. No presente trabalho, estudamos a fertilidade de ratas prenhes adultas tratadas com medicamentos anti-retrovirais isolados ou em associação. Os parâmetros analisados foram as taxas de perdas pré-implantação e eficiência de implantação (que refletem a quantidade de óvulos fertilizados e as perdas embrionárias anteriores à implantação endometrial) e as taxas de perdas pós-implantação e viabilidade fetal (que refletem as perdas embrionárias e fetais ocorridas após a implantação).

Quanto às taxas de TPP, não houve, no presente estudo, diferenças estatisticamente significativas entre os animais do grupo controle e aqueles tratados com medicamentos antiretrovirais, após 21 dias de prenhez. Em trabalho avaliando o efeito do AZT em ratas prenhes, Toltzis et al. ${ }^{12}$ observaram aumento das TPP com o uso desse anti-retroviral, demonstrando que o AZT interfere na divisão celular no embrião pré-implantado. Para Mamede et al. ${ }^{14}$, não houve elevação das TPP em ratas prenhes tratadas com AZT e aciclovir. Em casuistica semelhante, Fujinaga et al. ${ }^{15}$ também não demonstraram alterações nas TPP com utilização do AZT nos grupos de ratas prenhes tratadas, em comparação ao grupo controle.

Quanto às taxas de TEI, o presente estudo permitiu verificar que não houve diferenças entre os grupos de animais tratados com drogas antiretrovirais em relação às ratas do grupo controle no $21^{\circ}$ dia de prenhez. Resultados semelhantes são descritos por Toltzis et al. ${ }^{12}$ e Mamede et al. ${ }^{14}$, que não observaram alterações nas TEI de ratas prenhes tratadas com anti-retrovirais isolados ou em associação.

Quanto às TPPI, demonstrou-se que todos os grupos de ratas tratadas apresentaram TPPI com indices superiores àqueles observados no grupo controle. Sendo as TPPI complementares às taxas de TVF, todos os animais dos grupos que recebe- 
ram medicação apresentaram TVF abaixo dos niveis demonstrados no grupo controle. $\mathrm{O}$ estudo de Fujinaga et al. ${ }^{15}$ avaliou os efeitos do AZT, ddI, ddC e indinavir no período de prenhez de ratas. Estes fármacos foram utilizados de forma isolada e em associação, não sendo observadas alterações nas TPPI e TVF dos animais tratados em relação aos do grupo controle. Dos medicamentos utilizados na casuística citada, apenas o AZT foi utilizado no presente trabalho. Desse modo, os resultados não são plenamente comparáveis, justificando as diferenças encontradas.

Em 2000, Riecke et al. ${ }^{16}$ avaliaram os efeitos do indinavir durante o periodo de prenhez, não encontrando alterações nas TVF em ratas tratadas com esse inibidor de protease. Em estudo utilizando ddI, ddC e d4T em ratas prenhes, Toltzis et al. ${ }^{13}$ também não demonstraram repercussões negativas sobre as taxas de fertilidade dos animais expostos a esses fármacos. Como pode ser notado, as drogas utilizadas por tais autores diferem daquelas utilizadas em nosso estudo.

Observou-se também redução significativa do número de fetos por ninhada dos grupos de ratas tratadas, sendo que o peso dos fetos sofreu redução significativa nos grupos 3TC, AZT + 3TC e AZT $+3 \mathrm{TC}+\mathrm{NFV}$. O peso das placentas não sofreu alterações significativas com a utilização das drogas propostas.

Para Toltzis et al. ${ }^{13}$, Fujinaga et al. ${ }^{15}$ e Riecke et al. ${ }^{16}$, também não há diferenças quanto ao número e peso de fetos oriundos de ratas tratadas com medicamentos anti-retrovirais no período de prenhez. Entretanto, como já citado anteriormente, em nenhum desses estudos as combinações de drogas anti-retrovirais utilizadas se assemelharam àquelas utilizadas na presente casuística.

A despeito de não ser objetivo direto do atual estudo, a média de ganho ponderal diário das ratas prenhes tratadas com drogas anti-retrovirais e suas associações, demonstrou redução significativa nos grupos tratados com 3TC, AZT+3TC e $\mathrm{AZT}+3 \mathrm{TC}+\mathrm{NFV}$, após 21 dias de prenhez. Coincidentemente, os mesmos grupos tratados com 3TC apresentaram médias de peso fetal significativamente menores comparados à média de peso dos fetos do grupo controle. Em estudo utilizando aciclovir no período de prenhez, Mamede et al. ${ }^{14}$ observaram redução do ganho ponderal materno nos animais tratados com esse medicamento. Porém, após associação com AZT e nos grupos de animais recebendo apenas AZT, não houve redução significativa do ganho de peso materno.

Pelos dados expostos, pode-se notar que dentre os grupos de animais prenhes do presente estudo, aqueles nos quais o esquema de tratamento incluía o 3TC apresentaram redução significati- va do ganho de peso materno, bem como do peso fetal ao nascimento. Tal fato pode explicar a redução do ganho ponderal materno, uma vez que nesses grupos houve redução do ganho de peso fetal.

Adicionalmente, observou-se elevação das TPPI e conseqüente redução das TVF após 21 dias de prenhez em todos os grupos de animais recebendo anti-retrovirais isolados ou em associação, demonstrando que, provavelmente, os medicamentos utilizados durante a prenhez, nas doses propostas, induziram alterações importantes nos fetos após a sua implantação no endométrio, não ocasionando efeitos deletérios sobre a quantidade de óvulos fertilizados e tampouco sobre os embriões em periodo anterior à implantação. Essa afirmação pôde ser comprovada pela redução significativa do número de fetos por ninhada após 21 dias de prenhez nos grupos tratados com anti-retrovirais, em relação aos animais do grupo controle.

Alguns questionamentos podem ser levantados a partir desses resultados: o mecanismo de ação dos fármacos pode interferir nos processos bioquímicos de implantação e manutenção do embrião? A associação de drogas com mecanismos de ação diversos pode ocasionar um sinergismo de ações as quais podem prejudicar a circulação feto-placentária, impedindo o crescimento adequado do feto? Podem as drogas anti-retrovirais estar bloqueando algum processo bioquímicomolecular importante na fase de pós-implantação do embrião? Se algum desses fatores for verdadeiro, pode-se estender o raciocínio para humanos?

Os resultados e questionamentos advindos do presente estudo oferecem apoio à afirmação proposta por Riecke et $a .{ }^{16}$, que sugerem a realização de estudos para se avaliar a toxicidade reprodutiva de medicamentos anti-retrovirais isolados e em associação, de forma a determinar a segurança da utilização desses medicamentos no período gestacional.

\section{ABSTRACT}

Purpose: to evaluate experimentally the effects of antiretroviral drugs used alone and in association upon the fertility of pregnant Wistar rats and the perinatal effects on the offspring. Methods: adult female pregnant Wistar rats weighing 200$230 \mathrm{~g}$ were used. The antiretroviral drugs zidovudine (AZT), lamivudine (3TC) and nelfinavir (NFV) were used alone and in association at daily doses of ten times the dose normally used in pregnant women, proportionally to the animal's body weight. Seven groups were studied, including the control one. The experiment started on day 0 and the pregnant animals were sacrificed on day 21. The alive and dead fetuses, the total implantation sites and the total numbers of corporea lutea 
were used to calculate the fertility values. The statistical analysis was performed by Student's t test and by the MannWhitney test.

Results: there were no significant statistical differences regarding preimplantation loss and implantation efficiency values of the rats treated with isolated and associated antiretroviral drugs. There was a significant increase in the postimplantation loss values (control group: $7.6 \%$; drug groups variation: 20.2-26.7\%), a decrease in the fetal viability values (control group: 92.4\%, drug groups variation: 73.3$79.8 \%$ ), and a decreasing number of fetuses per animal (control group: 14.7; drug groups variation: 11.1-12.7). There was a significant weight reduction of the female rats and of the offspring of animals treated with $3 T C, A Z T+3 T C$ and $A Z T+3 T C+N F V$.

Conclusion: with the administration of high antiretroviral doses, important fertility effects could be observed, which showed that less histotoxic antiretroviral drugs must be studied in order to warrant the safety of using these medicines in pregnant HIV-1 - infected women.

KEY WORDS: Antiretroviral drugs. Infection and fertility. AIDS. Experimental study.

\section{Referências}

1. Connor EM, Sperling RS, Gelber R, et al. Reduction of maternal-infant transmission of human immunodeficiency virus type 1 with zidovudine treatment. N Engl J Med 1994; 331:1173-80.

2. Bulterys M, Lepage P. Mother-to-child transmission of HIV-1. Curr Opin Pediatr 1998; 10:143-50.

3. Lindegren ML, Byrs RH Jr, Thomas P, et al. Trends in perinatal transmission of HIV-1/AIDS in the United States. JAMA 1999; 282:531-38.

4. Stratton P, Mofenson LM, Willoughby AD. Human immunodeficiency virus infection in pregnant women under care at AIDS Clinical Trials Centers in the United States. Obst Gynecol 1992; 79:364-68.

5. Scott GB, Tuomala R. Combination antiretroviral therapy during pregnancy. AIDS 1998; 12:2495-97.

6. Martin R, Boyer P, Hammill H, et al. Incidence of premature birth and neonatal respiratory disease in infants of HIV positive mothers. J Pediatr 1997; 131:851-56.

7. Lorenzi P, Spicher VM, Laubereau B, et al. Antiretroviral therapies in pregnancy: maternal, fetal and neonatal effects. AIDS 1998; 12:F241-47.

8. Falcone A, Darnowski JW, Ruprecht RM, et al. Differential effect of benzylacyclouridine on the toxic and therapeutic effects of azidothymidine in mice. Blood 1990; 76:2216-21.

9. Ohnota H, Okada Y, Ushijima, H, et al. 3'-Azido-3'Deoxythymidine prevents induction of murine acquired immunodeficiency syndrome in C57BL/ 10 mice infected with LP-BM5 murine leukemia viruses, a possible animal model for antiretroviral drug screening. Antimicrob Agents Chemoter 1990; 34:605-9.
10.Ruprecht RM, Bernard LD, Chou TC, et al. Murine models for evaluating antiretroviral therapy. Cancer Res 1990; 50:5618S-27S.

11.Toltzis P, Marx CM, Kleinman N, et al. Zidovudineassociated embryonic toxicity in mice. J Infect Dis $1991 ; 163: 1212-18$.

12.Toltzis P, Mourton T, Magnuson T. Effect of zidovudine on preimplantation murine embryos. Antimicrob Agents Chemother 1993; 37:1610-13.

13.Toltzis P, Mourton T, Magnuson T. Comparative embryonic cytotoxicity of antiretroviral nucleosides. $\mathrm{J}$ Infect Dis 1994; 169:1100-2.

14. Mamede JA, Simões MDEJ, Novo NF, et al. Chronic effects of azidothymidine and acyclovir on pregnant rats. Gen Pharmacol 1995; 26:523-6.

15.Fujinaga M, Schulte M, Holodniy M. Assessment of developmental toxicity of antiretroviral drugs using a rat whole embryo culture system. Teratology 2000; 62:108-14.

16. Riecke K, Schulz TG, Shakibaei M, et al. Developmental toxicity of the HIV-protease inhibitor indinavir in rats. Teratology 2000; 62:291-300.

17.Ho RJ, Larsen K, Bui T, et al. Suppresion of maternal virus load with zidovudine, didanosine and indinavir combination therapy prevents mother-to-fetus transmission in macaques. J Acquir Immune Defic Syndr 2000; 25:140-9.

18.Petterson TA, Binienda ZK, Newport GD, et al. Transplacental pharmakocinetics and fetal distribuition of 2', 3'-didehydro-3'-deoxythymidine (d4T) and its metabolites in late-term rhesus macaques. Teratology 2000; 62:93-9.

19. Olivero AO, Fernandez JJ, Antiochos BB, et al. Transplacental genotoxicity of combined antiretroviral nucleoside analogue therapy in Erythrocebus patas monkeys. J Acquir Immune Defic Syndr 2002; 29:323-9.

20.Rondinini C, Venerosi A, Branchi I, et al. Long-term effects of prenatal 3'-azido-3'-deoxythymidine (AZT) exposure on intermale aggressive behaviour of mice. Psychopharmacology (Berl) 1999; 145:317-23.

21.Mamede JA, Oliveira-Filho RM, Simões MJ, et al. Hepatic and renal effects of azidothymidine and acyclovir on pregnant rats. Clin Exp Obstet Gynecol 2000; 27:227-30.

22.Venerosi A, Cirulli F, Lil'p IG, et al. Prolonged perinatal exposure to AZT affects aggresive behaviour of adult CD-1 mice. Psychopharmacology (Berl) 2000; 150:404-11.

23.Ricceri L, Venerosi A, Valanzano A, et al. Prenatal AZT or 3TC and mouse development of locomotor activity and hot-plate responding upon administration of the GABA(A) receptor agonista muscimol. Psychopharmacology (Berl) 2001; 153:434-42.

24.Venerosi A, Valanzano A, Alleva E, et al. Prenatal exposure to anti-HIV drugs: neurobehavioral effects zidovudine $(\mathrm{AZT})+$ lamivudine (3TC) treatment in mice. Teratology 2001; 63:26-37.

25.Almeida SA, Kempinas WG, Lamano Carvalho TL.Sexual behavior and fertility of male rats submitted to prolonged immobilization-induced stress. Braz J Med Biol Res 2000; 33:1105-9.

Recebido em: 18/11/2002

Aceito com modificações em: 27/ 12/2002 